\title{
The formation and preservation of chemical heterogeneities in the lower mantle
}

\author{
ANNA J.P. GÜLCHER ${ }^{1}$, JUN YAN ${ }^{1}$, MAXIM D. \\ BALLMER $^{213}$, PAUL J. TACKLEY ${ }^{1}$
}

${ }^{1}$ Department of Earth Sciences, ETH Zürich, Switzerland

${ }^{2}$ Department of Earth Sciences, UCL, London, UK

${ }^{3}$ Earth-Life Science Insitute, Tokyo Institute of Technology,

Tokyo, Japan

Despite stirring by vigorous convection over billions of years, the Earth's lower mantle appears to be heterogeneous on various length scales. On scales $<1 \mathrm{~km}$, the concept of a "marble cake" mantle has gained wide acceptance, emphasizing that streaks of recycled oceanic crust (ROC) and lithosphere make up much of the mantle. On larger scales (10s-100s of km), compositional heterogeneity may be preserved by delayed mixing of this marble cake with either intrinsically-dense or -strong materials of e.g. primordial origin. Intrinsically dense materials may accumulate as piles at the core-mantle boundary (CMB), while intrinsically viscous (e.g., enhanced in the strong mineral $\mathrm{MgSiO}_{3}$ bridgmanite) may survive as "blobs" in the mid-mantle for large timescales (i.e., as plums in the mantle "plum pudding"). So far, only few if any studies have quantified mantle dynamics in the presence of different types of heterogeneity with distinct strong+dense physical properties. Here, we address this objective using state-of-the-art 2D numerical models of global-scale mantle convection in a spherical geometry. We explore the effects of various rheological and physical parameters on mantle dynamics and mixing. Models predict that primordial heterogeneity is preserved in the lower mantle as discrete blobs for high intrinsic viscosity contrast $(>30 \mathrm{x})$. In turn, ROC tends to segregate from the marble cake mantle in regions of relatively low mantle viscosity. ROC is predicted to accumulate in the transition zone and the base of the mantle for a wide range of parameters. Importantly, these recycled crustal heterogeneities can co-exist with primordial blobs, suggesting that the modern mantle may be in a hybrid state between the "marble cake" and "plum pudding" styles. Convection models including preserved $\mathrm{MgSiO}_{3}$-enriched domains along with recycled piles have the potential of reconciling recent seismic observations of lower-mantle heterogeneity with the geochemical record from ocean-island basalts, and are therefore relevant for assessing Earth's bulk composition and long-term thermochemical evolution. 